\title{
INHALT DES ZWEITEN BANDES.
}

\section{Krankheiten der Luftwege.}

\author{
Krankheiten der Nase.
}

$\S$ 1. Hämorrhagie, Epistaxis, Nasenbluten

$\$$ 2. Der Katarrh der Nasenschleimhaut, Schnupfen, Coryza . . . . . . . . 3

a. Der acute Ǩatarrh, Coryza acuta.

b. Der chronische Katarrh, Coryza chronica u. Ozaena, Stockschnupfen $\mathbf{7}$

$\S 3$. Der Katarrh der Kehlkopfsschleimhaut.

a. Der acute Katarrh

b. Der chronische Katarrh

4. Der Croup, die häutige Bräune, Angina membranacea

5. Tuberkel und tuberculöse Geschwüre des Kehlkopfes $\quad . \quad 28$

\$ 6. Die syphilitischen Kehlkopfsleiden . . . . . . . . . . . . . . 31

7. Perichondritis laryngea, Larynxabcesse $\quad 33$

\$ 8. Oedema glottidis, Larynx-Oedem . . . . . . . . . . . . 35

9. Spasmus glottidis, Kehlkopfskrampf, Laryngismu stridulus . . . $\quad 37$

10. Paralysen des Kehlkopfs, Stimmbandlähmungen $\quad . \quad 40$

$\S 11$. Neubildungen im Kehlkopfe . . . . . . . . . . . . . . . . 44

Krankheiten der Pleura.

§ 1. Pleuritis, Brustfellentzündung .. . . . . . . . . . . . . . . 46

\$ 2. Hydrothorax, Brustwassersucht $\ldots 61$

3. Pneumothorax, Luftansammlung im Pleurasacke . $\quad 63$

\$ 4. Carcinom der Pleura. 67

§. Tuberculose der Pleura . . _ . . . . . . . . . . . 68

Krankheiten der Bronchen.

$\S$ 1. Bronchectasis, Bronchenerweiterung . . . . . . . . . . . . . . . . . 69

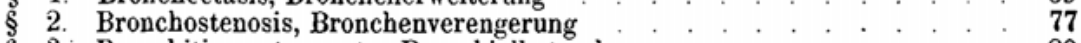

\$3. Bronchitis acuta, acuter Bronchialkatarrh $. \quad . \quad . \quad . \quad 80$

a. Der Katarrh der grösseren Bronchen $\quad . \quad 83$

b. Der Katarrh der kleineren Bronchen, Bronchitis capillaris . . . 84

$\S 4$. Bronchitis chronica, der chronische Bronchialkatarrh . . . . . . . . 89

a. Der einfache chronische Bronchialkatarrh . . . . . . . 92

b. Der chronische Bronchialkatarrh mit Degenerationen . . . . . 92 
a. mit emphysematöser Entartung des Alveolargewebes (L a ennec's Catarrhe sec).

p. mit Verdickung, Wulstung und papillären Wucherungen der Schleimhaut, Bronchoblennorrhoe

$\gamma$. mit Atrophie der Schleimhaut, Bronchorrhoea serosa

§ 5. Croup der Bronchialschleimbaut, Bronchitis crouposa . . . . . $\quad 97$

6. Tussis convulsiva, Keuchhusten, Stickhusten ‥ $\quad . \quad 999$

\$ 7. Asthma bronchiale, Bronchialkrampf, Spasmus bronchialis (R o m berg). 109

\section{Krankheiten des Lungengewebes.}

§ 1. Emphysema pulmonum, Lungenemphysem . . . . . . . . . . . 114

2. Atelektase, Luftleere der Alveolen . . . . . . . . . . . . . . 123

3. Hyperämie (Congestionen und Stasen) der Lungen . . . . . . . 126

4. Hämorrhagien der Athemwege, Haemoptysis, Haemoptoë, Bluthusten $\quad 133$

a. Bronchialblutungen _. . . . . . . . . . . . . . 133

b. Der hämorrhagische Infarct 138

c. Apoplexia pulmonum, Lungenschlagfluss . . . . . . . . 140

§ 5. Pneumonia crouposa, croupöse Lungenentzündung _. . . . . 141

§ 6. Die interstitielle Pneumonie . . . . . . . . . . . . . . . . 156

§ 7. Die katarrhalische Pneumonie . . . . . . . . . . . . . . 158

a. Die acute katarrhalische Pneumonie 159

b. Die chronische katarrhalische Pneumonie, käsige Pneumonie, scrophulöse Bronchopneumonie . . . . . . . . 163

§ 8. Die Tuberculose der Lungen . . . . . . . . . . . . . . . . . . 173

a. Die acute Miliartuberculose der Lungen . . . . . . . . . . 179

b. Die chronische Miliartuberculose der Lungen . . . . . . . . 181

§ 9. Der Lungenbrand, Gangraena pulmonum . . . . . . . . . . . . . . 187

\$ 10. Der Lungenkrebs

\section{Krankheiten der Geschlechtsorgane.}

§ 1. Pollutionen und Spermatorrhoe . . . . . . . . . . . . . . . 192

2. Die venerischen Krankheiten . . . . . . . . 195

a. Der Tripper, Gonorrhoe, Blennorrhoe der Harnö̈hrenschleimhaut 197

$\alpha$. Der Tripper des Mannes . . . . . . . . . . . . . 198

i) Der Tripper der Frau . . . . . . . . 210

Anhang: Spitze Condylome, Feigwarzen . . . . . . 211

211

c. Die Syphilis 217

a. Die syphilitischen Hautaffectionen, Syphiliden . . . $\quad 223$

Das maculöse oder erythematöse Syphilid . . . 224

Das papulöse Syphilid, Lichen syphiliticus, Knötchensyphilid 225

Das pustulöse Syphilid . . . . . . . . 227

Das tuberöse Syphilid, Hautgummata, Hautsyphilome (Wagner) . . . . 229

$\beta$. Die syphilitischen Schleimhautleiden $\quad 230$

$\gamma$. Syphilitische Erkrankungen einzelner Organe, in wie weit sie bisher nicht zur Besprechung gekommen sind . . . . . . . 232

Das Syphilom der Zunge . . . . . . . . 232

Die syphilitischen Erkrankungen der Nase . . . . 232

Die syphilitischen Erkrankungen der Muskeln . . . . . 233

Die Syphilis des Gehirns und seiner Häute ․ . . . . 234

Die syphilitische Erkrankung der Iris und Chorioidea . 236

Die syphilitische Hodenentzündung, Orchitis syph., Sarcocele syphilitica . . . . . . . . 237

Die syphilitischen Erkrankungen der Knochen und des Periost 238

Anhang: Syphilis congenita s. hereditaria. . . . . . 248 
Krankheitender weiblichen Geschlechtsorgane.

§ 3. Hyperänic. IIämorrhagie und Entzündung der Ovarien . . . . . . . 250

a. puerperale und b. extrapuerperale Oophoritis . . . . 251

\$ 4. Geschwülste : Cysten, Carcinom und Fibroide im Ovarium

$\$ 5$. Katarrh des Uterus und der Scheide, Endometritis catarrhalis.

a. im nicht puerperalen Zustande.

$$
\text { a. Der acute Katarrh . . . . . . . } 260
$$

per chronische Katarrh, Fluor allus, Leukorrhoe $\quad 262$

b. im puerperalen Zustande, End ^metritis puerperalis . . . . 265

$\$$ 6. Metritis parenchymatosa, Entzündung der Gebärmuttersubstanz.

a. Im nicht puerperalen Zustande.

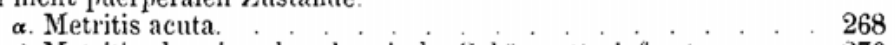

i. Metritis chronica, der chronische Gebärmutterinfarct . $\quad 270$

b. Im puerperalen Zustande, Itetritis und Parametritis puerperalis . $\quad 273$

\$ 7. Geschwüre an der Vaginalportion . . . . . . . . . . 277

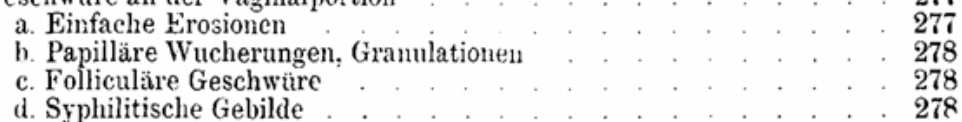

c. Das phagedänische Geschwir. Ulcus corrodens, ,Corroding ulcer of the os uteri"Clarke's . . . . 279

\$ 8. Hydrometra und Haemometra . . . . . . . . . . . . . . 279

9. Haematocele periuterina s. retrouterina . . . . . 281

$\$ 10$. Perimetritis . . . . . 284

\$11. Menstruations-Anomalien . . . . . . . . 288

a. Amenorrhoe 289

b. Dysmenorrhoe, Mienstruatio difficilis. Colica menstrualis . 292

«. Die nervöse Iysmenorrhoe. $\quad . \quad 292$

B. Die mechanische und organische Dysmenorrhoe _. . . 292

$\gamma$. Die membranöse Dysmenorrhoe $\quad . \quad 293$

c. Menorrhagie und Metrorrhagie 294

\$ 12 . Perperale IIämorrhagien.
a. Früh- und Spätblutungen . . . . . . . . . . 295

b. Die Placentarpolypen, đie tibrinösen Uteruspolypen, freie polypöse

Haematome des Uterus 297

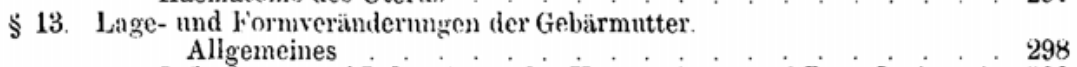

a. Inflexionen und Infractionen des Uterus (Ante- und Retroflexionen) 299

b. Versionen (Ante- und Retroversionen). 304

c. Yorfall der Gebärmutter und der Scheide, Prolapsus uteri et vaginae 306

\$14. Geschwülste des Uterus.
a. Das Myom, die fibromusculäre (ieschwulst, das Fibroid . . . . . 308
b. Die Schleimpolypen 311
c. Die Tuberculose
d. Das Fibrosarcom und das weiche, diffuse Sarcom . . . . 314
e. Das Carcinom und Cancroid
Anhang: Das Puerperalfieber . . . . . . . . . 317

\section{Vergiftungen.}

§ 1. Die Bleivergiftung . . . . . . . . . . . . . . . . . . . . . 321

a. Die acute 321

b. Die chronische $\quad 322$

a. Die Bleikachexie $\quad 32$

3. Die Bleikolik, Colica saturnina 322

$\gamma$. Affectionen von Rückenmarksnerren $\quad 323$

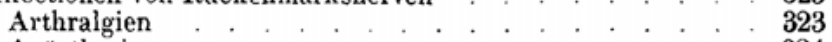

Anästhesien . . . . . . . . . . 324 
§ 2. Die Phosphorvergiftung . . . . . . . . . . . . . . . . . . 325

3. Die Arsenikvergiftung . . . . . . . . . . . . . . . . . . . . . . . . . 329

4. Die Alkoholvergiftung ～. . . . . . . . . . . . . . . . . . 333

a. Die acute . . . . . 334

b. Das Delirium tremens

c. Der Alkoholismus chronicus, die Säuferdyskrasie . . . . . . 339

\section{Infectionskrankheiten.}

\section{A. Infectionendurch Thiergifte, Zoonosen.}

§ 1. Lyssa, Wuthkrankheit . . . . . . . . . . . . . . . . . . 342

2. Milzbrand-Carbunculosis des Menschen . 348

§. Rotzkrankheit des Menschen, Malleus humidus et farciminosus, Equinia 351

B. Infectionen durch rein miasmatische Krankheitsgifte.

§ 4. Die Malariakrankheiten . . . . . . . . . . . . . . . . . . 356

a. Einfache Intermittenten . . . . . . . . . . . 360

b. Larvirte Intermittenten $\quad 366$

c. Perniciöse Intermittenten ․ . . . . . . . . . . . . . . 367

d. Remittirende Intermittenten . . . . . . . . . . . . . . 370

C. Infectionendurch miasmatisch-contagiöse Krankheitsgifte.

\$ 5. Die Cholera asiatica . . . . . . . . . . . . . . . . . . . . . . 373

§. Die Diphtheritis, Rachenbräune . . . . . . . . . . . . . . . 392

7. Die typhösen Erkrankungen . . . . . . . . . . 398

a. Der exanthematische Typhus, das Fleckfieber . . . . . . . 400

b. Der Ileotyphus, Abdominaltyphus, das Typhoid . . . 406

c. Der Typhus recurrens, Relapsing fever, Rückfallfieber . . . . 434

Griesinger's biliöses Typhoid . . . . . . . . . . . . 438

§ 8. Die Ruhr, Dysenterie . . . . . . . . . . . . . . . . . . 440

D. Infectionendurch die specifischen Contagien der sog. acuten Exantheme.

§ 9. a. Scharlach, Scarlatina . . . . . . . . . . . . . . . . . . 440b

b. Masern, Morbilli . . . . . . . . . . . . . 451

c. Rötheln, Rubeola . . . . . . . . . . . . . 458

d. Pocken, Blattern, Variola und Variolois $\quad . \quad$. . . . . 459

e. Windpocken, Wasserpocken, Varicellen . . . . . . . . . 469

f. Febris miliaris, das weisse Friesel, der englische Schweiss $\quad . \quad 469$

\section{Allgemeine Ernährungsstörungen, welche nicht von einer Infection abhängen.}

§ 1. Chlorose, Bleichsucht . . . . . . . . . . . . . . . . . . . . . 473

§ 2. Scorbut, Scharbock 479

3. Morbus maculosus Werlhofii, Blutfleckenkrankheit, Purpura haemorrhagica 490

4. Hämophilie, Bluterkrankheit . . . . . . . . . . . . . 405 
§ 5. Scrophulosis . . . . . . . . . . . . . . . . 495

6. Diabetes mellitus, Zuckerruhr $\quad 505$

7. Polyurie : Diabetes insipidus und Hydruric . . . . . . . 516

8. Rheumatismus $\quad 517$

a. acuter, Rheumatismus febrilis $\quad 519$

b. chronischer $\quad 531$

c. Der Muskelrheumatisnus $\quad 534$

$\alpha$. Der Rheumatismus der Kopfimuskeln und Kopfschwarte, Cephalalgia rheumatica. 536

3. Der Rheumatismus der Halsmuskeln, Torticollis rheumaticus 536

$\because$ Der Rheunatismus der Brustmuskeln, Pleurodynia rheumat. $\quad 537$

j. Der Rheumatismus der Bauchmuskeln, Rheumatismus abdominalis

8. Der Rheumatismus der Lendenmuskeln, Lumbago rheumatica

Der Rheumatismus der Extrenitätenmuskeln

$\$ 9$. Arthritis nodosa s. deformans, deformirende Gelenkentzündung, Malum senile

§ 10. Arthritis, Gicht, Podagra

13. Osteomalacie, Knochenerweichung $\quad 563$

\section{Krankheiten der Haut.}

$\S$ 1. Hautentzündungen, welche wesentlich in einer Hautröthung bestehen.

a. Erythema

b. Erysipelas, Rotl! !auf, Rose

c. Roseola

$\$$ 2. Hautentzündungen, bei denen sich Papeln bilden, die in ihrem ganzen ferneren Verlaufe keine weitere Ausbildung erlangen, sondern als solch e bestehen bleiben und dann ihre Rückbildung erfahren.

a. Lichen . . . . . . . . . 573

a. Lichen scrophulosorum . . . . . . . . . 573

B. Lichen ruber $\quad 574$

b. Prurigo, Juckblätterchen $\quad 575$

$\S 3$. Hautentzündungen, welche $B$ läs chen mit wässrigem Inhalt - Vesiculae - bilden.

a. Herves

Herpes labialis

Herpes praeputialis s. progenitalis . $\quad 577$

Herpes Zoster (Gürtelflechite) . . . . . . . . . . 577

Herpes Iris 578

Herpes circinatus (Willan) . . . . . . . . . . . 578

b. Ekzema, nässende Flechte $\quad . \quad 578$

Ekzema capillitii . . . . . . . . . . . . . . . 579

Ekzema faciei

Ekzema marmmae .

Ekzema genitalium . . . . . . . . . . . 580

Ekzema extremitatum . . . . . . . . . . 580

Ekzema marginatum $\quad 580$

§ 4. Hautentzündungen, welche grö ssere Blasen - Bulla $\mathrm{e}-$ bewirken.

a. Pemphigus, Blasenausschlag . . . . . 583

b. Rupia, Schmutzflechte $\quad 595$

§ 5. Hautentzundungen, welche Blasen mit ei trigem - Inhalt - Pustula e - bewirken.

a. Impetigo, nässender Grind (Achor) . . . . . . . . . . . 565

b. Ekthyma 586

§ 6. Hautentzündungen, welche Plättchen von abgestorbener Oberhaut, krankhafte Epidermisbildung - Squa ma e - bewirken. 
\begin{tabular}{ll} 
a. Pityriasis & 587 \\
\hline & 587
\end{tabular}

Pityriasis simplex . . . . . . . . . . . . 587

Pityriasis rubra . . . . . . . . . . . . . . . . . . . . . . . . . 588

b. Psoriasis, trockene Schuppenflechte . . . . . . . . . . . . . . . 588

c. Ichthyosis, Fischschuppenkrankheit _. . . . . . . . . . . . 591

$\S$ 7. Hautentzündungen, welche $\mathrm{Q}$ uad deln $-\mathrm{Pomphi}-$ bewirken. $\quad 593$

§ 8. Hautapoplexien, Hauthämorrhagien _. . . . . . . . . . . . . . . . $\quad .594$

$\S$ 9. Lupus, fressende Flechte

a. simplex . . . . . . . . . . . . . . . . . . . . . 596

b. erythematodes . . . . . . . . . . . . . 597

§ 10. Lepra, Aussatz, Elephantiasis Graecorum . . . . . . . . . . . . . . . $\quad$. 599

\$ 11. Elephantiasis Árabum . . . . . . . . . . . . . . . . . . . . . 601

§12. Sycosis, Mentagra, Bartflechte . . . . . . . . . . . . . . . . . . . . . . . . .603

a. simplex . . . . . . . . . . . . . . . . . . . . . . . . . . . . 603

b. parasitica . . . . . . . . . . . . . . . 604

§ 13. Krankheiten der Talgdrüsen. $\quad 605$

b. Milium, Comedo, Acne

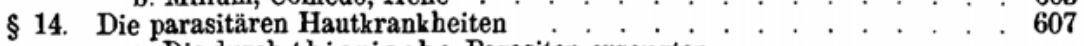

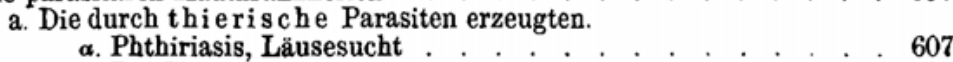

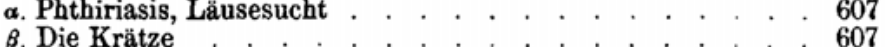

b. Die durch p flanzlich e Parasiten erzeugten.

a. Der Favus . . . . . . . . . . . . . . . . . . . . . . . . . . . . . . 611

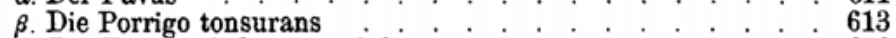

. Die Tinea pelada, Area Celsi . . . . . . . . . . . . . . . . . . . . . 616

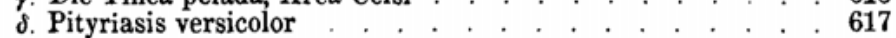

